\title{
MECHANICAL DESIGN ISSUES ASSOCIATED WITH MOUNTING, MAINTENANCE, AND HANDLING OF AN ITER DIVER'TOR*
}

\author{
P. L. Goranson, F. J. Fogarty, and G. H. Jones \\ Martin Marietta Energy Systems, Inc. \\ P.O. Box 2009 \\ Oak Ridge, TN 37831-8055
}

\section{Abstract}

Several designs that address plasma-facing plate configurations and thermal-hydrauiic design issues have been developed for the ITER divertor. Tesign criteria growing out of physics requirerents, physical constraints, and remote handling requiren :ents impose severe mechanical requirements on the support structure and its attachments. These pose a challenge to the mechanical design of a divertor, which must be addressed before a functional divertor is practical-that is, one that can be remotely handled, aligned, and maintained; that functions reliably under thermal loading and disruptions; and that gives the required life in the nuclear environment predicted for ITER.

This paper discusses the design criteria for the divertor mounting structure and identifies the mechanical design issues that need to be addressed. Achieving the criteria may require the development of new components and innovative configurations, specifically a new class of remote fasteners and electrically resistant material for mounts. The possible design of such components and an R\&D program to develop them are described, and issues specific to the high-aspect-ratio design (HARD) configuration are summarized. Analysis and experiments that will resolve these issuss and concerns and lead to a final ITER design are identified.

\section{Introduction}

For the plasma-facing divertor plate (DP) to be used during the physics phase of ITER, carbon-carbon monoblock on a copper substrate has been selected; for the technology phase, tungsten/niobium is a possible baseline design. For both phases, a modular (segmented) DP serves to diminish magnetic loads and provides a means for thermal expansion. This implies that a substructure must be used to preassemble the modules into a manageable number of units for installation. This structure fulfills multiple functions, as discussed below.

\section{DP Design Criteria}

Assessment of the proposed divertor concepts has led to the following design requirements.

\section{Mounting Structure}

The attachment method should be compatible with both physics and technology phases. The mounting structure should - provide an assembly base for divenor plates;

- react magnetic loads, both its own and that of the DP, meaning that it must have adequate strength and either be segmented and electrically isolated, to limit loops and eddy currents, or be electrically resistive;
- function as the remote handling structure (i.e., fit through the port, provide fittings for in-vessel manipulation, and be rigid enough to allow handling;

- provide a means for remotely aligning divertor modules;

- provide cooling circuits for itself and possibly the ISP;

- be dimensionally stable during thermal cycling of the system; and

- provide access to remote attachments.

Attachment

The means of attaching the structure to the vacuum vessel (VV) must be

- strong.

- electrically resistive to ground,

- remotely actuated, with no loose parts, and remotely accessible (it must be operated and adjusted through a small hole in the DP),

- easily disconnected in the event of failure, and

- compatible with thermal expansion and a nuclear environment.

\section{Baseline Design}

Two divertor support methods have been proposed for the baseline physics phase design. Both use thin flexible plates to attach the DP segments to substructure poloidal beams, either U-beams or I-beams, separated by gaps or insulation. These beams are connected by two stiff, water-cooled toroidal beams. The final configuration has its main support in the ends of the toroidal beams where it is atached to the vessel and an additional support at the cooling supply headers.

\section{Areas of Concem}

There are several areas of concerns with this design. First, disruptions are assumed to be toroidally symmetric. There is some concern with disruption distribution, particularly in the event of misalignment.

Large, poorly protected insulated surfaces can be expected to quickly metallize over and short. The resulting large conduction loops increase the loads severalfold. No suitable insulation material has bien identified; most exhibit significant degradation in performance under neutron bombardment.

Eddy currents manifest themselves as torsional loads about the poloidal axis of the divertor segments. This forces the beams to act as torsional members, an inefficient way to handle loads unless the uross section is a closed shape. Unfortunately, box members have large cross sections and high conductivity, which result in even larger loads. Also, the use of only three discrete mount locations could involve a high degree of risk, since it leaves meter-length portions of structure unsupported and, in fact, cantilevered at the inboard

* Research sponsored by the Office of Fusion Energy, U.S. Department of Energy, under contract DE-AC05-84OR21400 with Martin Marietta Energy Systems, Inc. 
end. It has not been demonstrated that this is sufficient structure even for rormal loading, let alone for off-normal loads that would occur in the event of insulation breakdown.

The baseline design proposes tapered cleats for retaining the divertor assembly to the vessel. The assembly would be slipped in under the cleats and the outboard end welded to the coolant headers. Unfortunately, there is no provision for tolerance buildup, the arrangement does not allow alignmert, and it could result in gaps at some locations, a condition that would result in impact loading of the structure.

No method for remote alignment of the DP segments has been developed. In addition, methods need to te developed for remote removal of divertors if there are problems with the attachments (e.g., cold-welding, stripped or galled threads, disruption damage).

\section{Proposed Solutions}

\section{Mounting Structure}

An altemative design would build on the design proposed for the technology phase, replacing the many segmented pieces tied together by insulators (or oxide layers). Instead, it may be possible to design a large, integrated structure, which significantly reduces disruption loads by careful manipulation of geometry and is strong enough to tolerate the temaining loads. Such a geometry is shown in Fig. 1.

The structural mount is a one-piece ribbed plate that can accommodate DP segments of either physics or technology phase design (Fig. 2). The configuration uses a "Christmas tree" design with slots cut toroidally in the plate face. The slots break up the surface area and reduce the eddy current loads. They also block halo currents and confine them to the small header regime up the centerline of the plate. The geometry has not been modeled, but hand calculations are encouraging (see Fig. 3) and indicate that survival seems possible.

Ribs running toroidally have several advantages. First, they allow simple fabrication, since the ribs remain plane and do not have to be formed. The entire assembly is machined in the flat and then hydroformed to the final contour. The design also allows a very simple cooling circuit that feeds water out of the central headers into the ribs as branch loops (Fig. 1). This results in a temperature rise of only $30^{\circ} \mathrm{C}$ anywhere in the structure. The combination of poloidal ribs on the DP bolted to toroidal ribs on the mount plate results in a structure that is very rigid and easy to handle. The configuration also reduces the electrical cross section in the toroidal direction and further reduces distuption current.

\section{Structure Attachment}

Load management: A transverse mounting scheme is proposed. It eliminates the torsional component and allows the structure to act as a series of independent beams simply supported on each end. The beam can then have an open section with small poloidal cross section and easier access to fasteners.

DP altachment: Individual DP segments are bolted to the substructure from the underside so that no access holes are required in the front face of the DP. Holes or slots may be left in edges of the DP between segments so that two units each share half of the hole. The resulting space is $19 \mathrm{~mm}$ wide; all work must be accomplished in this space. Two approaches are presented here for using this $19-\mathrm{mm}$ access hole; an expanding lug, as shown in Fig. 4, and a conventional entrapped remote cone-head bolt, as shown in Fig. 5.

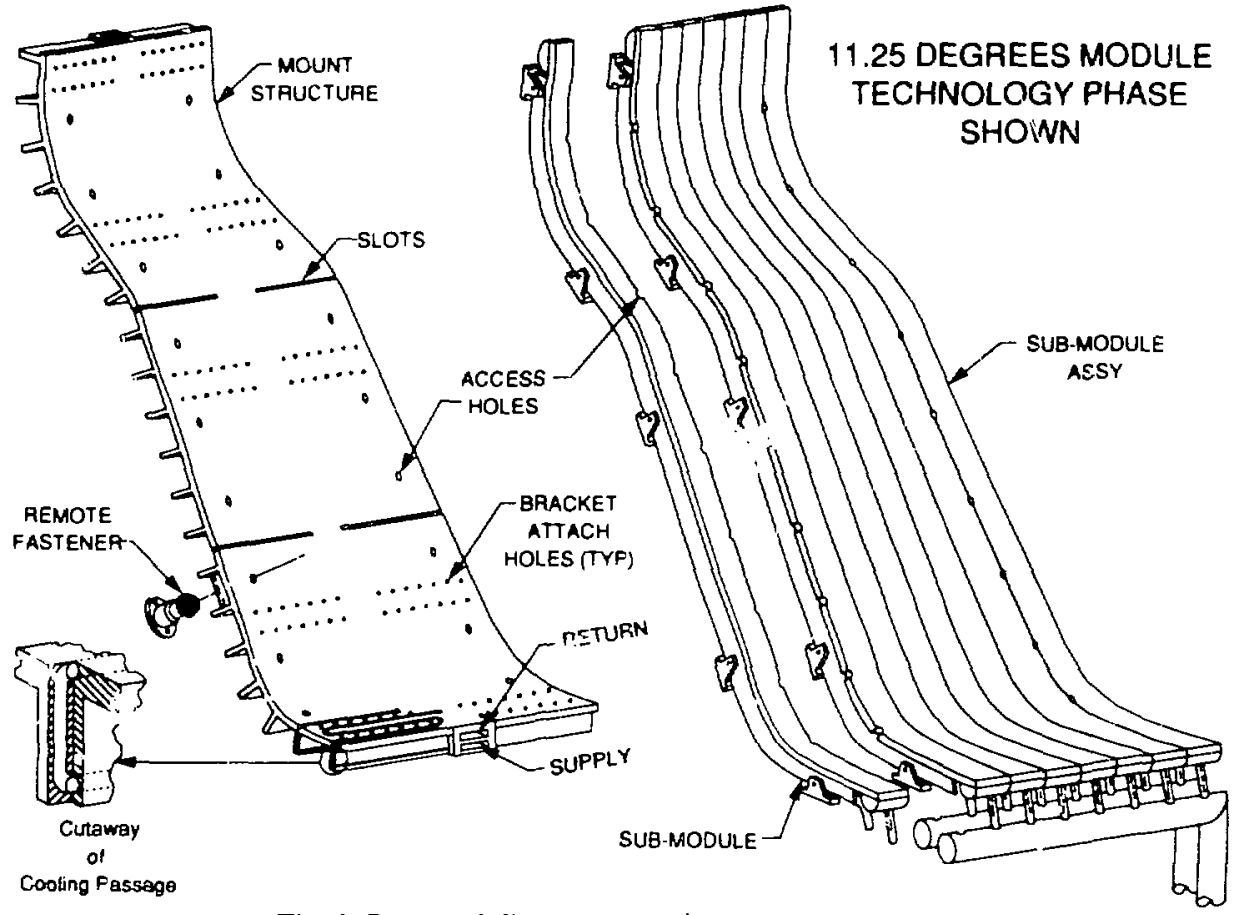

Fig. 1. Propored divertor mounting structure. 


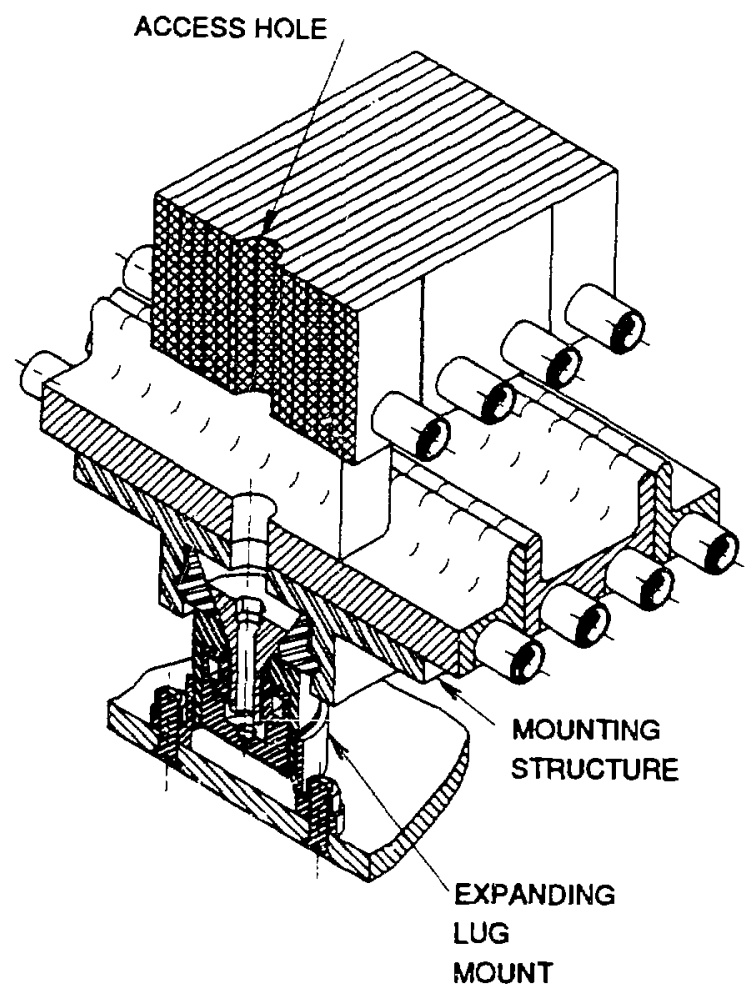

Fig. 2. Mounting structure (physics phase).

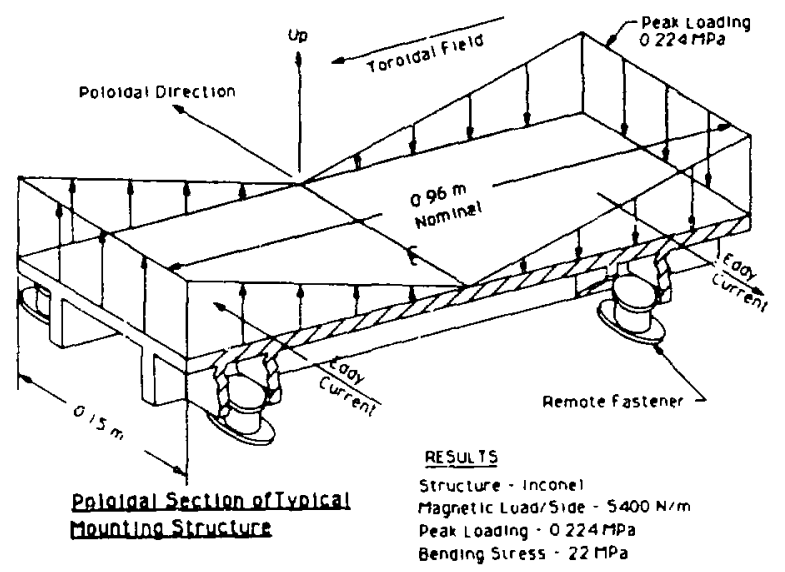

Fig. 3. Magnetic loads on proposed divertor mounting structure.

The expanding lug is preattached to the vessel shielding and is self-contained; no parts must be handled by the in-vessel manipulator other than a drive tool. The divertor assembly is placed on guide pins, the drive tool is inserted through the DP access hole to engage the lug, the inner hex drive is turned to adjust the lug until alignment is achieved, and finally the outer hex drive is tumed to expand the lug until it engages the ribs in the structural mount plate. The adjustable base of the lug could incorporate resistive material, as described below.

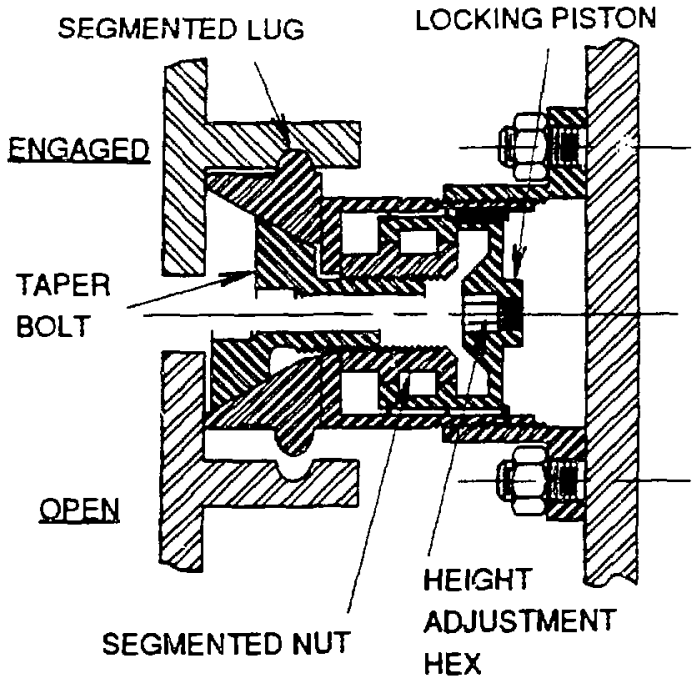

Fig. 4. Expanding lug concept.

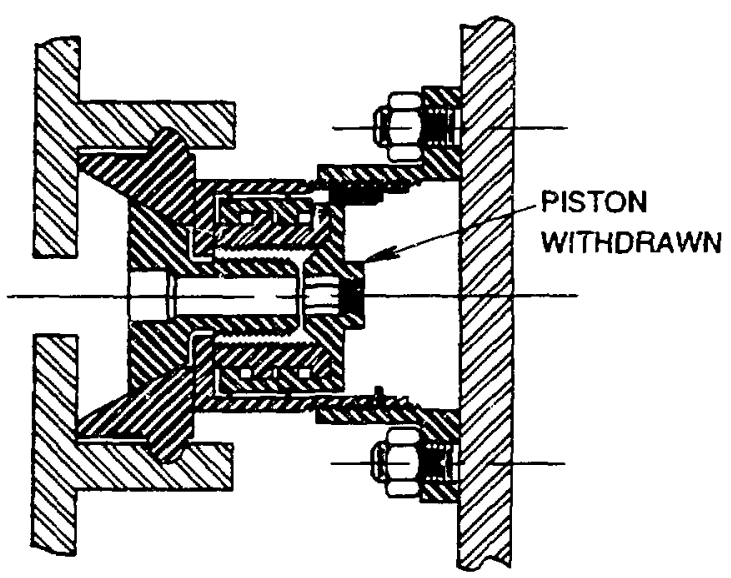

Fig. S. Conventional cone-head bolt concept.

During initial operation the lug can be disengaged and readjusted at will; later, after exposure to vacuum, heat, and radiation, the lug may freeze and not disengage. Therefore, the lug is provided with a special breakaway feature (Fig. 6). A segmented nut retains the lug. A pull tool can be inserted into the hollow lug to engage the sliding piston that retains the nut. When actuated, the piston will release the nut segments and drive them apart, thus releasing the lug from its base. A variation on this design is a breakaway nut with a calibrated "weak poine," a groove that shears when overtorqued. Preliminary thermal calculations indicate that the lug temperature will stay within a workable limit. Heat loss is via conduction to the water-cooled diveror structure.

A conventional remote, rctained bolt can be used with the access holes provided (Fig. 5). It is compatible with the breakaway nut design as well. 
DIVERTOR PLATE

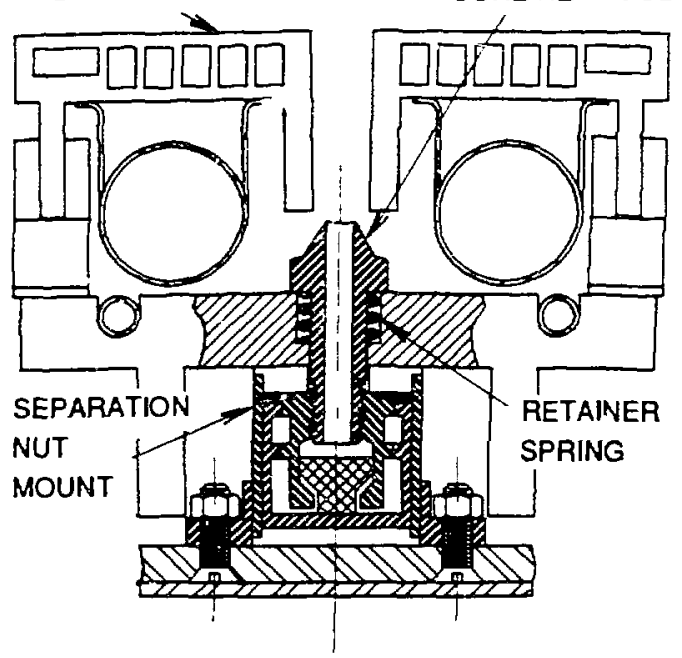

Fig. 6. Release of bolt.

\section{Electrically Resistive Components}

High resistance to ground (a few milliohms) between the divertor assembly and the vessel is necessary. A resistive coupling could significantly reduce damage during disruptions by limiting current and at the same time prevent the arc-over that is prone to occur at gaps ana insulated surfaces. Unfortunately, even Inconel and graphite are not resistive enough, indicating that a new family of materials for diventor mounts should be invesugated.

Desirable characteristics for such a material include

- high resistance (custom designed),

- high strength and malleability,

- ease of fabrication,

- versatility (weldable, machinable), and

- low thermal stresses.

Two candidates are sintered composites (Cermets) and diffusion-bonded barrier resistors. No suitable Cermet has been found by the authors in literature searches to date. Most lack the required malleability and must be treated as ceramics. Several potential candidates for diffusion bonding have been identified. The Soviets have developed an extensive database on bonding ceramics and semiconductors to metals and have made low-stress resistive bonds with the following: ${ }^{1}$

\begin{tabular}{lll}
\hline Ceramic & Base material & Interstitial \\
\hline $\mathrm{Si}$ & Molybdenum & Nickel \\
$\mathrm{Ge}$ & Nichrome & Silver \\
$\mathrm{SiC}$ & Kovar & Gold \\
& Invar & Aluminum \\
\hline
\end{tabular}

A possible design configuration for a diffusion-bonded resistive mount is shown in Fig. ?.

\section{Impact On HARD}

The divertor configuration is essentially unchanged for the HARD design. The segmentation will undoubtedly be somewhat different, but this would only have a minor effect on divertor mounting--perhaps a slight shift in fastener location but no change in methodology. Clearance for handling and installation in the divertor region is decreased, however, and this may have a impact on the divertor mounting. ${ }^{2}$

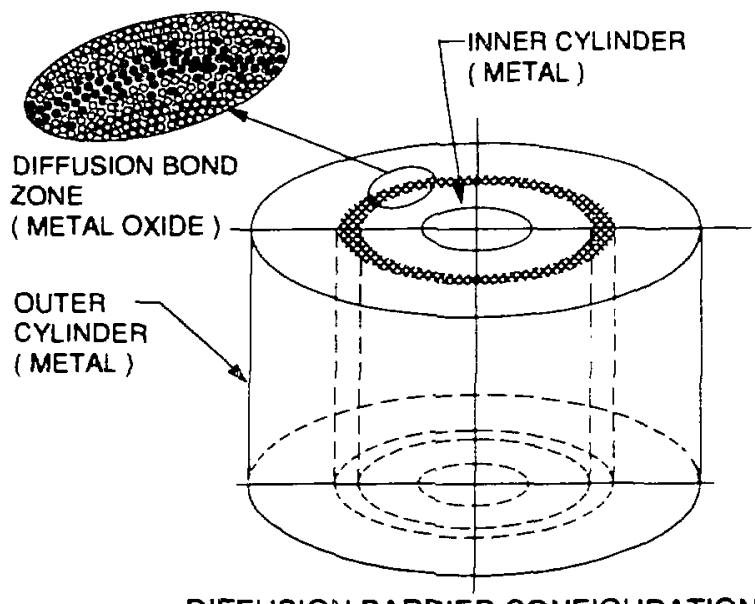

DIFEUSION BARBIER CONFIGURATION

- DIFFUSION ZONE IS TRANSITION FROM METAL TO OXIDE.

- DIFFUSION ZONE THICKNESS IS VARIED BY TIME, TEMP, PRESSURE AND ORIGINAL OXIDE THICKNESS.

Fig. 7. Resistive mount.

\section{Unresolved Areas}

- Feasibility of remore installation of fasteners.

- Survival of divertor suructures. Disruptions must be better characterized and accurate magnetic loads calculated.

- Divertor installation tolerances. Thermal loads will dcpend on the actual location of the divertor relative to the plasma.

\section{Analysis and R\&D Tasks Proposed}

- Magnetic load modeling of the divertor mounting structure.

- Thermal modeling to determine stresses and distortion of the structure.

- Modeling and optimization of the effects of resistive mounts.

- Thermal analysis of the expanding lug fastener.

- Construction of prototype fasteners and structural tests.

- Evaluation of resistive materials and development of prototypes.

\section{Conclusions}

The baseline divertor structure is dependent on insulators to limit magnetic loads; these insulators have not been defined and are vulnerable to shoring. A suitable, remotely installed attachment scheme for the divertor has not been designed.

An integral mounting structure that does not require insulators may be possible. This structure could be attached by remotely installed fasteners that incorporate a new family of resistive materials. The designs proposed are very preliminary, but initial results are encouraging and further development is warranted.

\section{References}

[1] N. F. Kazakov, Diffusion Bonding of Materials. New York: Pergamon Press, 1985, ch. 15, pp. 257-274.

[2] F. C. Davis et al., "Effect of High Aspect Ratio on ITER Maintenance Design," these proceedings. 